Journal of Applied Fluid Mechanics, Vol. 15, No. 2, pp. 311-324, 2022.

Available online at www.jafmonline.net, ISSN 1735-3572, EISSN 1735-3645.

https://doi.org/10.47176/jafm.15.02.32337

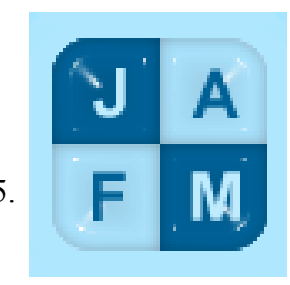

\title{
Turbulence Structures in Accelerated Flow over a Flat Plate with Non-Zero Pressure Gradient
}

\author{
M. Kharghani and M. PasandidehFard ${ }^{\dagger}$ \\ Department of Mechanical Engineering, Ferdowsi University of Mashhad, Mashhad, 91775-1111, Iran \\ †Corresponding Author Email: fard_m@um.ac.ir
}

(Received October 31, 2020; accepted June 6, 2021)

\begin{abstract}
The numerical simulation of temporally accelerated flow subjected to Favorable and Adverse Pressure Gradients (FPG \& APG) is represented. Two accelerations are considered and imposed on fully turbulent flow over a flat plate. The $\gamma$-SST model is implemented to define the boundary layer development, turbulence structures, and the wall functions responses to the external accelerated flow. The obtained results illustrate that the FPG condition accompanied by temporal acceleration can severely damp the fluctuations. So, an almostlaminar boundary layer develops near the wall, followed by a retransition to the higher turbulent state. However, the APG condition provides higher turbulence diffusion in a temporal accelerated flow. It makes the flow more orderly and more stable, although a re-laminarization is observable in this region excessively. Moreover, the applied condition disturbs the Reynolds stress correlation and generates semi-elliptic eddies because acceleration affects wall-normal fluctuations more severely than the streamwise ones. Also, a new represented acceleration parameter for this condition is compared with the acceleration parameter in spatially accelerated flow.
\end{abstract}

Keywords: Turbulent flow; Unsteady flow; Boundary layer; Pressure gradient; Turbulence kinetic energy; Spatially acceleration.

\section{NOMENCLATURE}

$\begin{array}{ll}c_{f} & \text { skin friction coefficient } \\ k & \text { turbulence kinetic energy } \\ K & \text { acceleration parameter } \\ K_{t} & \text { acceleration parameter in temporally } \\ & \text { accelerated flow } \\ L & \text { plate length } \\ R e & \text { Reynolds number } \\ R e_{\delta} & \text { Reynolds number based on the } \\ & \text { boundary layer thickness } \\ t & \text { time } \\ t^{*} & \text { non-dimensional time } \\ T & \text { acceleration period } \\ T u & \text { turbulence intensity } \\ u^{\prime} & \text { perturbation velocity in x-direction }\end{array}$

\section{INTRODUCTION}

Turbulent boundary layers are subjected to variable pressure gradients in many engineering and environmental applications. Turbulent flow in some applications could be exposed to both temporal and spatial acceleration; such as the reduction (or widen)

$\begin{array}{ll}U_{0} & \text { initial velocity } \\ U_{I} & \text { final velocity } \\ v^{\prime} & \text { perturbation velocity in the } \mathrm{y}- \\ & \text { direction } \\ x & \text { streamwise coordinate } \\ y & \text { wall normal coordinate } \\ \text { Greek symbols } \\ \delta(x, y, t) \quad \text { boundary layer thickness } \\ \theta(x, y, t) \quad \text { momentum thickness } \\ \rho & \text { fluid density } \\ \vartheta & \text { viscosity } \\ \vartheta_{t} & \text { turbulent viscosity } \\ \tau_{\mathrm{w}} & \text { wall shear stress }\end{array}$

of the river bed width, while flow is essentially unsteady.

Combining these two circumstances causes complex phenomena that are not comprehensively understood as well as the canonical zero-pressure-gradient boundary layers. The reason relies on much wider parameter space and the difficulty in determining universal scaling laws similar to those of the zero- 
pressure-gradient cases. Both temporal and spatial accelerations display unique features examined in various research. However, the primary emphasis of this paper is on these two specific circumstances' interaction. This paper tries to explore the dynamics of these processes that are fewer addressed by other researchers.

\subsection{Spatially accelerated flow}

Spatially accelerated flow is an interesting subject for many researchers. The favorable and adverse pressure gradients are sort of this condition. As a result of the Favorable Pressure Gradient (FPG), turbulence becomes less sustained. According to a process called 're-laminarization' or 'quasilaminarization,' the phase of the flow exchanges to a quasi-laminar state. To assess these conditions, an acceleration parameter $K=\left(\vartheta / U_{\infty}^{2}\right) d U_{\infty} / d x$ is proposed, where $x$ is the streamwise direction, $U_{\infty}$ is the free-stream velocity in this direction, and $\vartheta$ is the kinematic viscosity. This parameter evaluates the acceleration level in the FPG condition.

There are various investigations regarding the relaminarization mechanism. Spalart (1986) showed that if parameter $\mathrm{K}$ exceeds the value of $2.5-3 \times$ $10^{-6}$ in the sink flow (the equilibrium accelerating boundary layer that occurs in a long converging duct with straight walls), re-laminarization started, and turbulence cannot sustain. Narasimha and Sreenivasan (1973) conducted that reversion in highly accelerated flows is essentially caused by the domination of pressure forces over the slowly responding Reynolds stresses in an originally turbulent flow, followed by the generation of a new laminar boundary layer stabilized by the FPG. Through an experimental investigation by Narayanan and Ramjee (1969), they attributed that the wall zone adjusts itself to the laminar conditions before the outer zone and around the $\operatorname{Re} \simeq 300$, where $\mathrm{Re}$ is free-stream Reynolds number, the turbulence intensity starts to decline. Blackwelder and Kovasznay (1972) examined the large eddy structure during the re-laminarization in a strongly FPG condition. They showed that Reynolds stresses remain constant along the streamline in the outer wall region. Whereas the intermittency factor decreased along the streamlines until the turbulence level reaches its minimum in most of the boundary layer. Ichimiya et al. (1986) observed low-amplitude intermittent fluctuations of the velocity in relaminarization, and irregular fluctuations with high amplitude that appear gradually in retransition.

There are also various proposed criteria for relaminarization. Narasimha and Sreenivasan (1973) concluded that the re-laminarization results from the pressure gradient domination against the Reynolds stress and make them "Frozen" in the outer layer. Besides, the Reynolds shear stresses maintain their correlation in the acceleration region. More recently, McEligot and co-workers (1983, 2006, 2009) examined the flow in a laterally converging duct. They observed that the burst frequency is susceptible to acceleration. A strong pressure gradient affects the transport of momentum and reduces it in the viscous layer's outer part.
Experimental investigations focused on the high and low-pressure gradient are done by Warnack and Fernholz (1998). Their studies were in various accelerations between $K=0$ and $K=4 \times 10^{-6}$ and different Reynolds numbers. Intense accelerations cause an increment in the streamwise Reynolds stresses in the near-wall region, while they initially decrease and then begin increasing in the outer region. The shear stresses demonstrated a decrease and an increase, afterward.

Re-laminarization and the laminar-like phase of the boundary layer engaged with a significant change in the behavior of near-wall turbulence quantities. Escudier et al. (1998) also presented hot-wire measurements in a converging channel. They claimed that in the boundary layer's near-wall region, the turbulence intensity scales roughly with the freestream velocity up to close to the location where $k$ is a maximum, whereas the outer region $u^{\prime}$ remains nearly frozen. Another empirical study is devoted to Bourassa and Thomas (2009). They examined the FPG generated by a linear contraction, established that the inner layer is more sensitive to the acceleration than the outer layer, and turbulent production and Reynolds-stress correlation are affected strongly and decreased during the relaminarization process.

Following these studies, Scotti and Piomelli (2002) performed a Large Eddy Simulation (LES) of a spatially developing boundary layer with two freestream velocity distributions. In comparison between two cases with the sufficient and insufficient pressure gradient to re-laminarization, the streaks become more elongated in the near-wall region and illustrate fewer undulations, relating to a significant decrease of the spanwise fluctuations relative to the streamwise ones. As the flow becomes more instantaneous, they concluded that the vortexstretching and re-orientation due to the streamwise velocity gradient is the primary mechanism driving by increased dissipation near the wall and more intense vortices. They performed another research using the LES and Direct Numerical Simulation (DNS) (Piomelli and Yuan 2013) and showed that the pressure fluctuation reduction damps the streamwise stresses. They also proposed that $v^{\prime}$ and $w^{\prime}$ are damped because of the energy redistribution reduction while $u^{\prime}$ does not decay. In another study, Yuan and Piomelli (2015) examined this phenomenon on the rough wall using the DNS method to describe the sequences more precisely. It is ascertained in their study that these two conditions are coupled, as the accelerated flow on the rough wall leads to higher turbulence production and higher wake intensity, which causes a higher pressure drag and higher transfer between the wake's energy and that of the turbulence. According to that, Bader et al. (2018) implemented experimental and numerical research using $\gamma-R e_{\theta}$ turbulence model for the numerical part and their focuse was on the relaminarization and re-transition of the turbulent boundary layer. More recently, Saavedra et al. (2020) compared DNS and RANS calculation using the $k$ - $\omega$-SST model in free stream accelerated flow over a flat plate. They observed that RANS models 
could capture the dynamic sequences in bypasstransition conditions. Besides, imposed acceleration affects the shear stress and heat transfer because of the stretch in both momentum and thermal boundary layer profiles.

\subsection{Temporal accelerated flow}

There are two specific types of unsteady turbulent flow: periodic pulsating flows and non-periodic transient flows. In the present study, the uniform non-periodic transition flows are discussed. One of the early studies about such flows is devoted to Mizushina et al. (1975), who used the electrochemical method through an experimental investigation in pulsating pipe flow. They observed that there is a time delay of the turbulence propagation in the center of the pipe. Following this way, Shemer and Kit (1984), Shemer et al. (1985), and Burnel et al. (1990) have studied such flows through experiments using hot wires. These researches conducted that the effects of velocity and frequency factors on the speed and vibration of turbulence fields in pipes are considerable. Still, the impact of the amplitude is not significant.

One major research of the uniform acceleration and deceleration of transient flows in a pipe devotes to He and Jackson (2000), who employed the Laser Doppler Velocimetry (LDV). They instanced three main delays in response to the turbulence production, energy, and turbulence radially distribution in the turbulence field. Well ahead, this study has been numerically followed by Jung and Chung (2009) using Large Eddy Simulation (LES) with the well confirmation of previous experimental results. Greenblatt and Moss (2004) conducted a similar experimental study of transient turbulent pipe flow. They identified that the imposed pressure gradient in the higher flow rate leads to a freezing phase of the turbulent regime in the core region of the pipe and initially characterized the velocity profile, which reconstructed at the final phase of the acceleration. In another study, He et al. $(2008,2011)$ examined the influence of turbulence and inertia on wall shear stresses in transient turbulent pipe flow both experimentally and numerically. They reported three stages of shear stress development: the frozen stage, slowly increasing and dropping to its pseudo steady value

In addition to the mentioned researches on pipe flow, there are various researches on temporally accelerated channel flow. He and Seddighi (2013) studied a rapid increase in the flow rate of the turbulent channel flow employing the DNS method. They indicated that turbulent flow exposed to the sharp acceleration undergoes a process resembling the laminar boundary layer's bypass transition. They also measured this case by varying the initial and final Reynolds number ratios ( $\mathrm{He}$ and Seddighi 2015). Their results displayed that the bypass-liketransition phenomenon in the large increase of the Reynolds number is more remarkable than the low Reynolds number ratios, while some other flow structures, like the transition scenario and the streamwise fluctuating velocity, are comparable in both conditions.
Furthermore, they compared the DNS results of the initially turbulent channel and pipe flow in the same conditions (He et al. 2016). They revealed that the behavior of near-wall flow structures in pipe and channel is similar, and pipe flow exhibits a transition scenario as well. Jung and Kim (2017) observed other flow parameters relevant to the temporally accelerating channel flows' transition behavior by applying the DNS method and Reynolds number ratio's variation. Mathur et al. (2018) also manipulated a laboratory experiment on flow accelerated from an initially turbulent state following a valve opening operation combined with the LES of their experiments and extended Stokes's first problem solutions for the early stages of the flow.

Despite a large variety of researches in internal (pipe and channel) temporally accelerated flow, there are not sufficient investigations focused on external temporally accelerated flows, which is the main emphasis of the present paper. One of the earliest experimental studies on uniform accelerating flows belongs to Sarpkaya, which applied to a circular cylinder and significantly simplified the theoretical formulation (Sarpkaya 1991). Also, Freymuth (1985) has also made an experimental parametric study on accelerating flow passing a NACA0015 airfoil at the different angles of attack from $20^{\circ}$ to $90^{\circ}$ to investigate the complexity of vortex patterns and structures. The more recent experimental study is devoted to Soria et al. (2003) on $30^{\circ}$ angle of attack NACA0015 airfoil exposure to uniform accelerated flow using PIV (MCCDPIV) method. They indicated that the shear layer's intensity and the vertical structures are highly intense at higher accelerations. Sengupta et al. (2007) investigated the accelerated flow with $\mathrm{Re}=7968$ under different accelerations both experimentally and numerically.

In the present study, the T3C5 flat-plate test bench is set up to examine the temporally accelerated flow with a variable pressure gradient. Despite the decades of research, some of the mechanisms and structures involved in temporally accelerated flow with changes in pressure gradient is still unclear. The above researches are devoted to spatially or temporally accelerated flow. But, the combination of these two conditions is fewer examined by researchers. Besides, most of the studies on temporally accelerated turbulent flow are devoted to pipe and channel flow. The purpose of this research is to examine the effect of the pressure gradient on temporally accelerated flow in an open channel, in not only the re-laminarization mechanism but also turbulence production rate after the breakdown period. In addition, in real applications, the positive and negative pressure gradients are imposed subsequently. Thus the effect of these two conditions on each other is one of the main subjects addressed in this manuscript.

The following section presents the numerical setup involved in the numerical validation, the acceleration criteria used in this study, and the appropriate turbulence model selection. Afterward, it presents and discusses the measurement results focused on transport phenomena and turbulence structures, 
focusing on the re-laminarization process. In the final section, the paper ends with some concluding.

\section{NUMERICAL METHOD}

The European Research Consortium on Flow, Turbulence, and Combustion (ERCOFTAC) T3C5 series in the case of the transitional boundary layer on a flat plate with favorable and adverse pressure gradient imposed by the opposite converging/diverging slip wall and the experimental data of ERCOFTAC (Http://Cfd.Mace.Manchester.Ac.Uk) database is considered in this paper. The present research attempts to access the most similarity for the computational domain with an existing experimental test case.

Fig. 1 represents the computational domain of the considered flat plate with a variable pressure gradient. The domain consists of $\mathrm{L}=2$ meter long wall at the bottom and an upper slip wall with variable height. At the inlet section, the gap between the upper and lower walls is $0.3 \mathrm{~m}$. This distance adjusts along the flow path corresponds to the experimental data of pressure gradient variance. The computational domain starts at $0.15 \mathrm{~m}$ upstream from the plate leading edge to prevent the creation of a natural point of stagnation and its effect on the boundary layer. In Fig. 1, the parameter $\mathrm{H}$ represents the height of the input domain. The amplitude profile of the upper wall for the T3C5 geometry is defined by Eq. (1), where $x$ is the distance from the leading edge of the plate. Also, on the left and right of the domain, the velocity inlet and pressure outlet are applied, respectively.

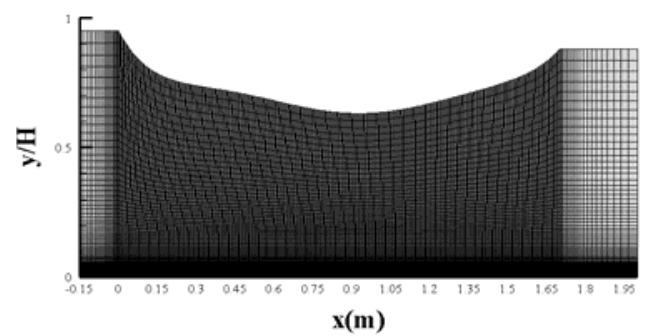

Fig. 1. Computational domain and mesh distribution for the transitional boundary layer on TC5 flat plate.

The computational domain's exact geometry, which presents the pressure gradient proportional to the experimental data, can be obtained using continuity and knowing the velocity at each cross-section. The fluid around the plate is considered incompressible with specified constant values of the density and molecular viscosity equal to $998.2 \mathrm{~kg} / \mathrm{m}^{3}$ and $1 \times 10^{-6}$ $\mathrm{kg} / \mathrm{m} . \mathrm{s}$, respectively.

$$
y / H=\operatorname{Min}\left[\begin{array}{l}
1.23 x^{6}-6.705 x^{5}+14.06 x^{4} \\
-14.113 x^{3}+7.109 x^{2} \\
-1.900 x+0.950 ; 1
\end{array}\right]
$$

\subsection{Acceleration scheme}

In analyzing the external accelerated flows, two different approaches are considered. The first and simpler one is to consider the body as the fixed object while fluid starts to flow around that by variable momentum. Another methodology is to assume that the flow accelerates around the stationary body. The second assumption in an experimental setup is applied by fixing the solid body and making the flow rate by moving a piston to access the physical accuracy and avoid failure caused by sharp transition loads. Moreover, this method provides significant simplifications in mathematical formulations of the problem and can validate various numerical methods (Sengupta et al. 2007).

The present work tries to investigate the turbulence structures in various flow rates and pressure gradients. This case is considered as a fundamental and practical example of different engineering and environmental applications such as humans blood flow, open channels with changing in width (e.g. river bed), and flow around the solid objects.

The inlet velocity follows a linear equation to access the most similarity in simulation, defining the acceleration. As demonstrated in Eq. (2), the velocity in a moment can be calculated as a function of initial $\left(U_{0}=0.571\right)$ and final velocity $\left(U_{1}=5.571\right)$ and the acceleration period $(\mathrm{T})$

$$
U_{t}=\frac{U_{1}-U_{0}}{T} t+U_{0}
$$

\subsection{Computational validation}

According to the previous results, just two extreme accelerations $\left(10 \mathrm{~m} / \mathrm{s}^{2}\right.$ and $\left.20 \mathrm{~m} / \mathrm{s}^{2}\right)$ are examined because of the similarity of milder accelerations response to steady-state flow. In this case, the initial velocity is $0.57 \mathrm{~m} / \mathrm{s}$ which levels up to the value of $5.57 \mathrm{~m} / \mathrm{s}$ under the mentioned accelerations. The static pressure decreases initially from 6 Pascal to 193 Pascal, which reduces to about $0.9 \mathrm{~m}$ from the beginning of the plate, then increases continuously to -32 Pascal. The details of the acceleration periods are presented in Table 1. Accordingly, $\mathrm{T}$ is the period when the initial velocity reaches its final amount under the considered acceleration, and $\Delta T$ is the time step considered for each acceleration period.

Table 1. Considered accelerating periods

\begin{tabular}{|c|c|c|c|}
\multicolumn{4}{|c|}{ details } \\
\hline Case & $\mathrm{T}$ & $\Delta \mathrm{t}$ & $\mathrm{du} / \mathrm{dt}\left(\mathrm{m} / \mathrm{s}^{2}\right)$ \\
\hline $\mathrm{AC} 3$ & $0.5 \mathrm{~s}$ & $5 \mathrm{e}-6$ & 10 \\
\hline $\mathrm{AC} 4$ & $0.25 \mathrm{~s}$ & $25 \mathrm{e}-7$ & 20 \\
\hline
\end{tabular}

\subsubsection{Mesh independence study}

The computational domain shown in Fig. 1., contains fine-squared cells that are sufficiently scattered along the wall and the leading edge with a nonuniform distribution in the wall-normal direction and a uniform distribution along the streamwise direction. To ensure that the first cell is placed 
underneath the baffle layer, the $\mathrm{y}^{+}$of the first cell is kept under 0.9 . The mesh resolution is $800 \times 210$ cells in streamwise and wall-normal directions, respectively. They are scattered with 1 and 1.011 expansion coefficient and sufficiently makes a good agreement with the experimental results as presented in Fig. 2.

\subsubsection{Time-step independence study}

To examine the accuracy of numerical setup in transient simulation, time step size should be considered. Therefore, different time steps are examined in sharper acceleration. Table 2 shows that after the time step of $2.5 \mathrm{e}-6$, the wall friction coefficient does not represent a considerable change. In the case of $\mathrm{AC} 3$ item, time step has been adopted using the constant value of $\Delta t / T$.

Table 2. Details of the mesh and time step independencies

\begin{tabular}{|c|c|c|c|c|}
\hline Grid & cells & Case & $\Delta \mathrm{t}$ & $C_{f}$ \\
\hline G1 & 78000 & AC4 & $5 \mathrm{e}-6$ & 0.0251 \\
\hline G2 & 102400 & AC4 & $25 \mathrm{e}-7$ & 0.0243 \\
\hline G3 & 168000 & AC4 & $1 \mathrm{e}-5$ & 0.0271 \\
\hline G3 & 168000 & AC4 & $25 \mathrm{e}-6$ & 0.0233 \\
\hline G3 & 168000 & AC4 & $1 \mathrm{e}-6$ & 0.0221 \\
\hline G3 & 168000 & AC4 & $25 \mathrm{e}-7$ & 0.0210 \\
\hline G3 & 168000 & AC4 & $1 \mathrm{e}-7$ & 0.0209 \\
\hline
\end{tabular}

\subsection{Turbulence model study}

Aiming to ensure that the selected model is appropriate for the present transient simulation, the turbulence model validation is studied in two steps: firstly, steady-state validation of turbulent model; secondly, validation of the model with experimental data of accelerated flow over the hydrofoil NACA0015. Accordingly, three turbulence models are considered: transient SST, $\gamma-\mathrm{SST}$, and $\mathrm{K}-\mathrm{K}_{\mathrm{L}}-\omega$ models. Fig. 2 compares the wall friction coefficient using mentioned turbulence models with the experimental data. As shown, the $\gamma$-SST model agreed well with the experimental data in predicting the transition region, whereas two other models do not indicate that precisely. However, as the simulation in this paper is transient, it requires careful consideration in this specific condition. Fig. 3 demonstrates that the present simulation is in good agreement with Sengupta et al.'s (2007) experimental results around the NACA0015 hydrofoil. The Couple method is employed to coupling the pressure and velocity transport equations. A second-order upwind scheme and the finite volume discretization for all equations are employed.

Khaleghi et al. (2010) compared different turbulence models to analyze an accelerating channel flow and showed that the SST model has a good agreement with DNS data in near-wall regions. The $\gamma$-SST model, which solves only one equation, also can predict the re-laminarization in highly streamwise acceleration conditions (Menter et al. 2015). Therefore, this model may be an appropriate choice to solve the accelerating flow equations by strictly envisaging the transition and re-laminarization, especially for the present test case.

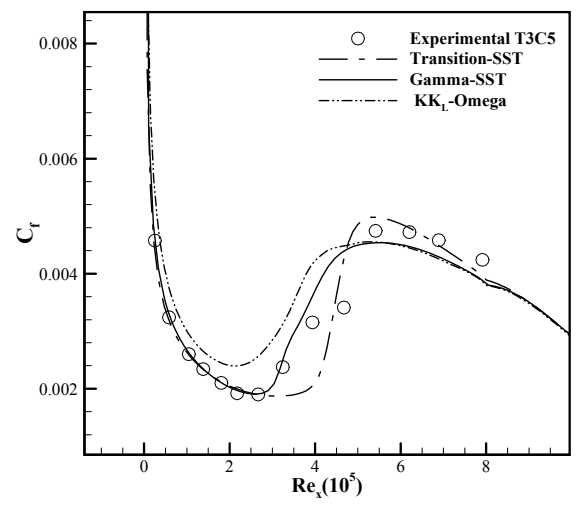

Fig. 2. Comparison of wall friction coefficient in three different turbulence models with experimental results in $\mathrm{T3C} 5$ case.

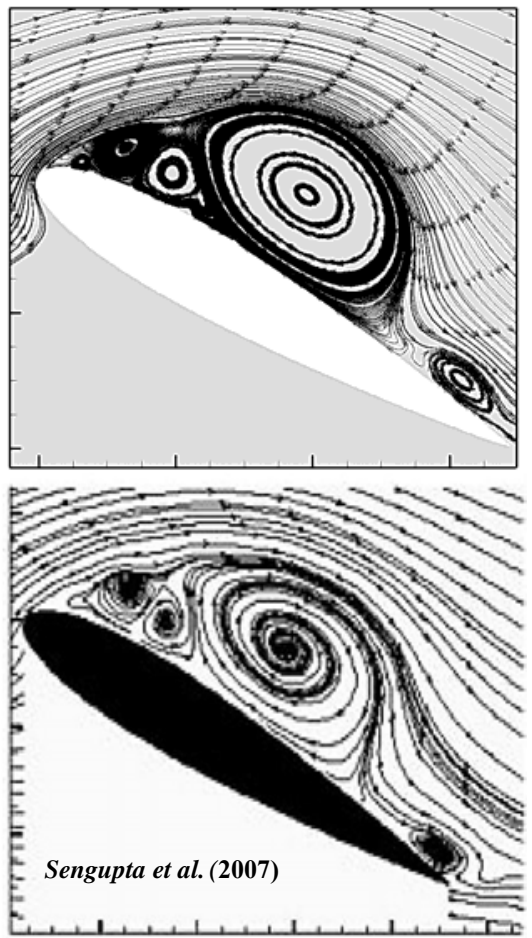

Fig. 3. Comparison of experimental results of Sengupta et al. (2007). (below) and present simulation (up) of accelerating flow over NACA0015 airfoil.

\subsubsection{Transport equations}

It is necessary to solve a dimensionless form of the momentum and continuity equations for all Newtonian's incompressible flow as following:

$$
\frac{\partial u_{i}}{\partial x_{i}}=0
$$


M. Kharghani and M. PasandidehFard / JAFM, Vol. 15, No. 2, pp. 311-324, 2022.

$$
\frac{\partial u_{i}}{\partial t}+u_{j} \frac{\partial u_{i}}{\partial x_{j}}=-\frac{\partial P}{\partial x_{i}}+\frac{1}{\operatorname{Re}} \nabla^{2} u_{i}
$$

Equations (1) and (2) are the continuity and momentum equations, respectively, where $R e=$ $U x / v$ and $\mathrm{i}, \mathrm{j}=1,2$ for the streamwise and wallnormal directions.

In order to include the turbulence effect in the problem, the $\gamma$-SST model has been selected, which is coupled with SST two-equation model (Menter 1994) and solve one transport equation $\gamma$, which has a good agreement for transition prediction over the flat plate (Menter et al. 2015). This model is a further development of the $\gamma-R e_{\theta}$ model (Völker 2006), which avoids solving an extra formulation of $R e_{\theta}$, so reduces the computational effort; in addition, has a better agreement with experimental results as shown in Fig. 2. The details of this model is presented in Menter et al. 2015.

The transport equation for the intermittency is as the following:

$$
\begin{aligned}
& \frac{\partial(\rho \gamma)}{\partial t}+\frac{\partial\left(\rho U_{j} \gamma\right)}{\partial x_{j}}=P_{\gamma}-E_{\gamma} \\
& +\frac{\partial}{\partial x_{j}}\left[\left(\mu+\frac{\mu_{t}}{\sigma_{\gamma}}\right) \frac{\partial \gamma}{\partial x_{j}}\right]
\end{aligned}
$$

This equation can be coupled with the SST turbulence model and is accomplished by modifying the equations of the original SST model (Menter 1994) as follow:

$$
\begin{aligned}
& \frac{\partial}{\partial t}(\rho k)+\frac{\partial}{\partial x_{j}}\left(\rho u_{j} k\right)=\gamma P_{k}+P_{k}^{\lim }-\boxplus_{k} \\
& =\max (\gamma, 0.1) \cdot D_{k}+\frac{\partial}{\partial x_{j}}\left(\left(\mu+\sigma_{k} \mu_{t}\right) \frac{\partial k}{\partial x_{j}}\right) \\
& \frac{\partial}{\partial t}(\rho \omega)+\frac{\partial}{\partial x_{j}}\left(\rho u_{j} \omega\right)=\alpha \frac{P_{k}}{v_{t}}-D_{\omega} \\
& +C d_{\omega}+\frac{\partial}{\partial x_{j}}\left(\left(\mu+\sigma_{\omega} \mu_{t}\right) \frac{\partial \omega}{\partial x_{j}}\right)
\end{aligned}
$$

where $P_{k}$ and $D_{k}$ are the production and destruction terms from the turbulence kinetic energy equation in the original SST turbulence model (Menter 1994) and the $P_{k}$ term is computed using Kato-Launder formulation:

$$
P_{k}=\mu_{t} S \Omega
$$

Where $\Omega$ is the magnitude of the absolute vorticity rate. The $P_{k}^{l i m}$ term is an additional term to ensure proper generation of $k$ at transition points for arbitrary low (down to zero) $T_{u}$ levels. This term is designed to be zero when the boundary layer has reached the fully turbulent $\operatorname{state}(\gamma=1)$. This term is defined as follows:

$$
\begin{aligned}
& P_{k}^{\lim }=5 C_{k} \max (\gamma-0.2,1) \\
& \times F_{o n}^{\lim } \max \left(\left(3 C_{S E P} \mu-\mu_{t}\right), 0\right) S \Omega \\
& F_{o n}^{\lim }=\min \left(\max \left(\frac{\operatorname{Re}_{V}}{2.2 \cdot \operatorname{Re}_{\theta c}^{\lim }}-1,0\right), 3.0\right) \\
& \operatorname{Re}_{\theta c}^{\lim }=1100 \\
& C_{k}=1.0, C_{S E P}=1.0
\end{aligned}
$$

\section{RESULTS}

According to Fig. 4, the solution domain starts with a negative (favorable) pressure gradient and continues with an adverse pressure gradient (positive). In the following simulation, the boundary layer thickness is affected by the pressure gradient; also, the imposed momentum triggers instabilities over that.

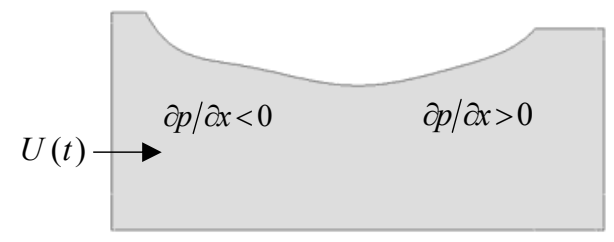

Fig. 4. T3C5 geometry with favorable and adverse pressure gradients.

\subsection{Wall functions}

It is crucial to predict the wall functions like friction coefficient in advance to track the flow behavior. According to previous researches, accelerated flow always accompanies a delay that prevents the fluctuating velocities from developing through the boundary layer. This delay becomes more remarkable in sharper accelerations. In other words, in more intense accelerations, there is a considerable time at which the turbulence intensity and kinetic energy do not change considerably while the main time average velocity increases significantly; so, the flow is considered as a freezing phase. Imposing momentum rate to the temporal accelerating flow could cause a pressure gradient over the flow domain, which resembles the FPG condition.

Skin friction coefficient $\left(C_{f}\right)$ versus nondimensionalized time $t^{*}=t / T$ at different regions is illustrated in Fig. 5. At the FPG region, $C_{f}$ experiences a fluctuating manner. The pressure drop intensifies delays at near-wall regions and prevents that location from synchronizing well with the outer layer Reynolds number. Thus, the duration of the time at which the flow is unconscious of the changes approximates twice the time delay in the APG region. 

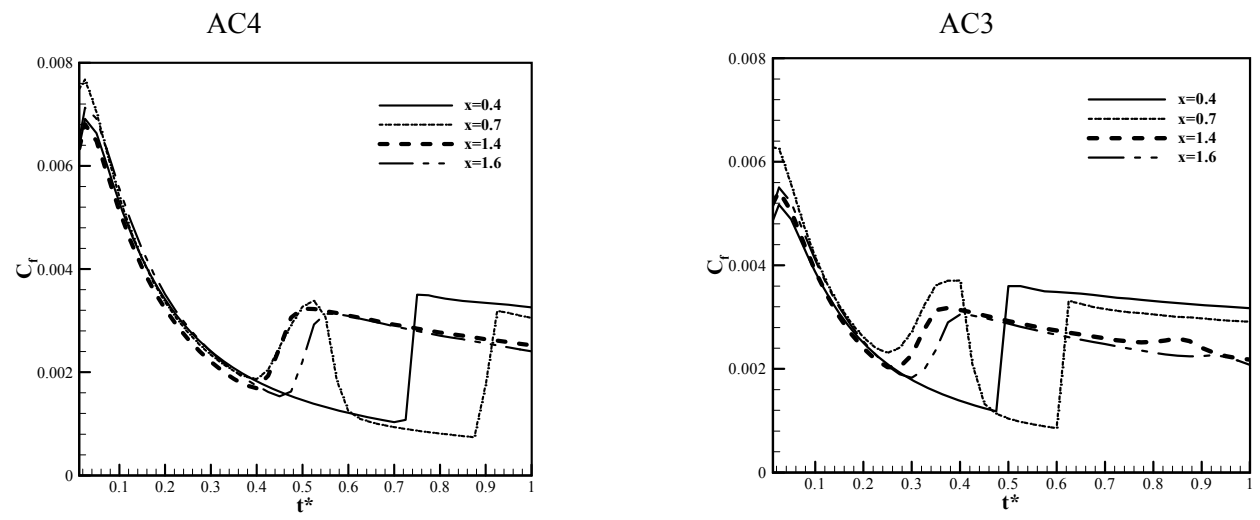

Fig. 5. Wall friction coefficient history in four specific streamwise positions regions for cases AC3 \& AC4.

Regarding the $\mathrm{x}<0.95$ section, at regions close to the minimum pressure, flow is exposed to more instabilities. This is further observable in $\mathrm{x}=0.7$ (Fig. 5.), where flow witnessed another re-laminarization and bypass transition and other regions. The reason for this behavior relies on the boundary layer interactions, which are discussed in the following.

In Fig. 6., the wall shear stress in different time zones, in the case of AC4, is compared with the steady-state condition. The wall shear stress under the acceleration condition is generally higher than the steady-state one. According to Fig. 6., the distinction of wall shear stress value between the steady-state flow and the accelerated one in the APG region is more significant, which means that the velocity profiles have a flatter shape compared to the FPG zone. Regarding the turbulence structure, as the wall friction increased, the $\mathrm{y}^{+}$ increased, meaning that the viscous forces that damp the fluctuations become weaken in this circumstance resulted in greater turbulence intensity.

\subsection{Boundary Layer Development}

Figure 7 compares the boundary layer thickness variations in zero and non-zero pressure gradient (T3C5) conditions in $20 \mathrm{~m} / \mathrm{s}^{2}$ acceleration and at the end of the acceleration period. Plus, the $R e_{\delta}$ (Reynolds based on the boundary layer thickness) variation for the $\mathrm{AC} 4$ test case is shown in this figure. For the zero pressure gradient case, the T3A ERCOFTAC test case $\mathrm{http} / / / \mathrm{cfd}$.mace.manchester.ac.uk/ercoftac) is selected, and the identical acceleration and boundary layer conditions are imposed to have the most effective comparison between these two conditions.

Adverse pressure gradient condition causes a dramatic decrease in boundary layer thickness at about $x=0.75$. However, the boundary layer can overcome the imposed momentum to some extent $(x \lesssim 0.7)$. After a while, turbulence fluctuations are damped drastically, and boundary layer thickness decreases sharply. However, having passed the FPG region, the boundary layer thickness mounts sharply.
The $\operatorname{Re}_{\delta}$ also decreased between $x=0.75$ to $x=0.95$, which shows that the turbulence instabilities decreased in that region, and the re-laminarization becomes probable.

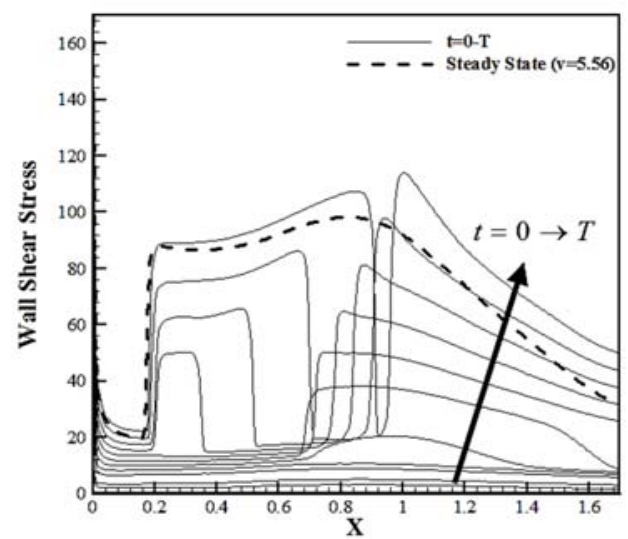

Fig. 6. Wall shear stress variation along the plate in various times in comparison with the steady-state.

The turbulence kinetic energy propagation in the AC4 case is demonstrated in Fig. . As it is displayed, soon after the acceleration commencement $\left(\mathrm{t}^{*}=0.075\right)$, turbulence propagation is affected severely. Consequently, a laminar boundary layer develops through the wall and transfers the flow to the so-called re-laminarization period.

As time passes and velocity increases, the turbulence fluctuations near the minimum pressure region $(x \cong$ 0.95 ) develops over the FPG area but do not exceed less than $x=0.65$. Indeed, after the freezing period $\left(t^{*}=0.35\right)$, the turbulence production rate at the minimum pressure region is high and disturbs its left and right sides. As the local velocity is frequently higher than the other areas in that region, this area is more potential to generate disturbances. 


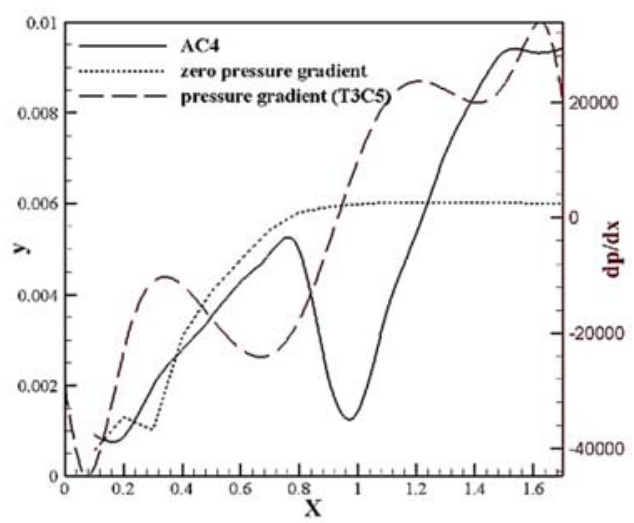

(a)

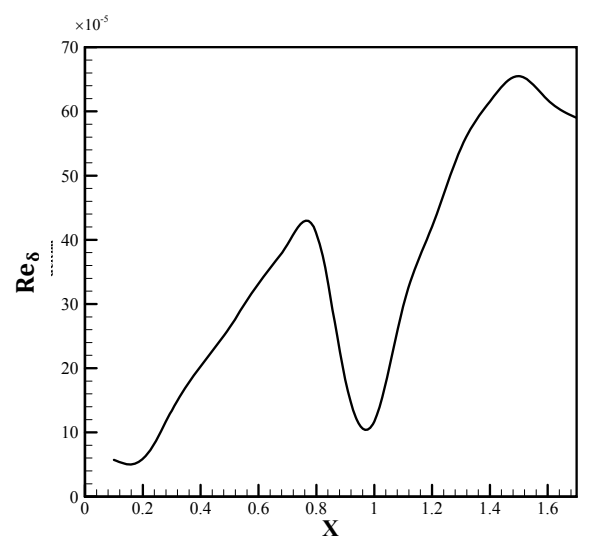

(b)

Fig. 7. (a): Boundary layer development in zero and non-zero pressure gradient in AC4 case and pressure gradient variation along the wall in $\mathrm{AC} 4$ case, $(\mathrm{b}): \llbracket \mathrm{Re} \rrbracket \_(\delta)$ variation along the plate (at the end of the acceleration period)
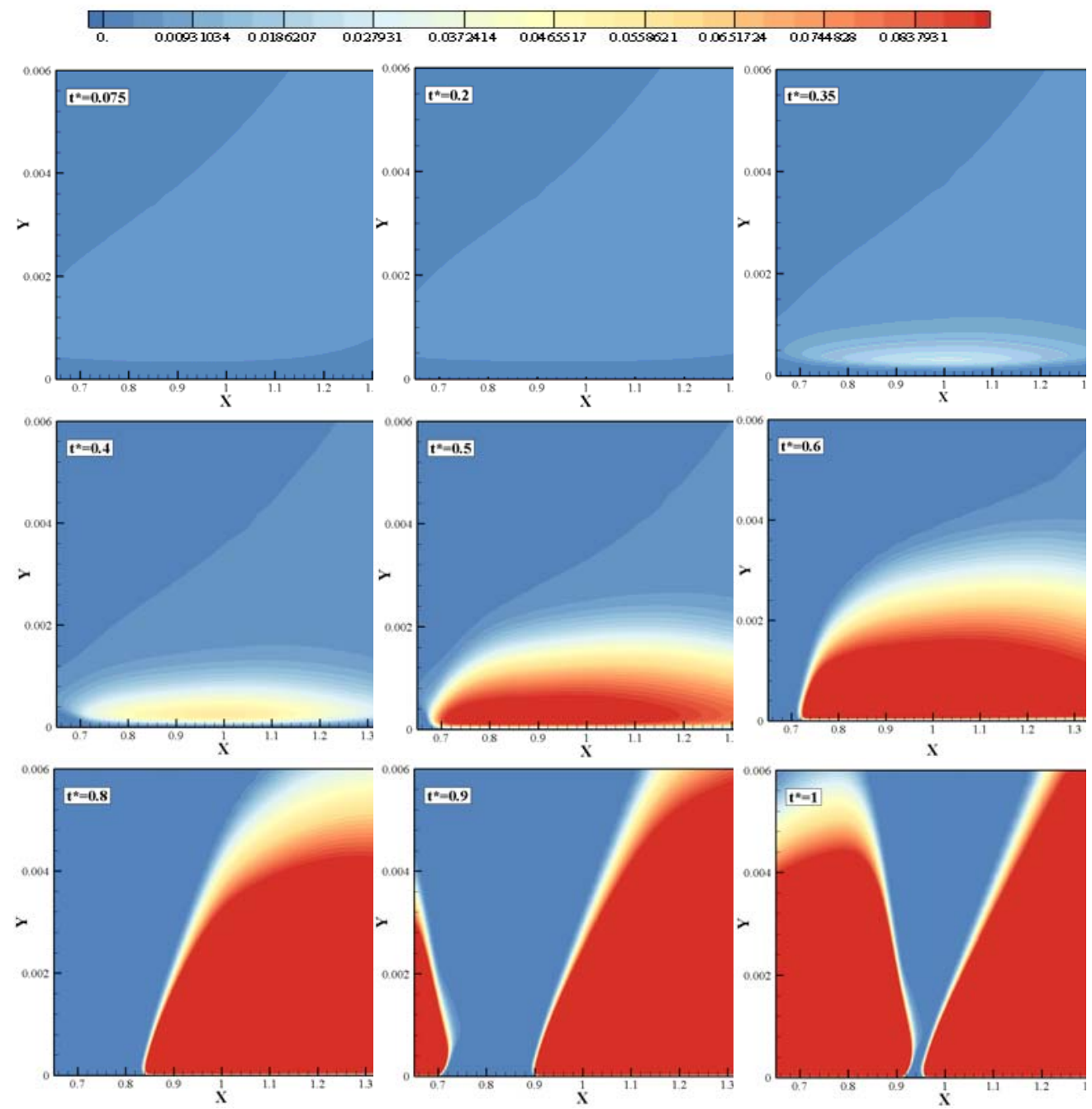

Fig. 8. Turbulence kinetic energy development history at $x=[0.65,1.35]$ in AC4 case. 
The boundary layer thickness $(\delta)$ decreases at the minimum pressure region, which causes a higher rate of fluctuating velocity transfer to the outer layer; thus, the delay duration reduces. In contrast, the negative pressure gradient induces an incensement in delay. However, as the local Reynolds number in the outer region increases considerably, reduced boundary layer thickness triggers the fluctuating velocities to spread out. Still, these disturbances do not sustain (Piomelli and Yuan 2013, Yuan and Piomelli 2015); the reason is discussed in detail in the following sections.

After the appearance of the disturbances at the minimum pressure region $\left(t^{*}=0.35\right)$, they start to dissipate at around $t^{*}=0.525$ from the right side $(x \leq$ $0.95)$. Subsequently, the FPG region becomes almost laminar. Indeed, the reduction of $\mathrm{Re}_{\delta}$ at that region causes this re-laminarization. Afterward, the fluctuations start to develop from the inlet of the plate, where the flow contains enough potential to overcome the exposed momentum.

On the other hand, due to the higher $\operatorname{Re}_{\delta}$ at the APG region, the fluctuations spread throughout the boundary layer from about $t^{*}=0.3$ to the end of the acceleration period.

\subsection{Re-laminarization Mechanism}

In certain conditions, such as spatial acceleration, the reverse transition from a turbulent to a laminar regime may occur. The main reason for this process is reducing the pressure fluctuations and, consequently, its effect on energy redistribution in Reynolds stresses in the initial turbulent state. Under such situations, it cannot be claimed that Reynolds' stresses have been disappeared. Then, it initiates almost frozen shear stress (Menter 1994). As a result, an almost-laminar boundary layer develops near the wall, characterized by the reverse of the turbulent velocity profile to the laminar one, a sharp decrease in the wall friction coefficient, and a reduction of the turbulence intensity. In previous researches, the acceleration parameter $K$ is represented by considering the spatial acceleration condition.

However, in the particular circumstance of this study, the acceleration factor $K_{t}$ is represented as Eq. (13), which considers both streamwise and temporal accelerations.

$$
K_{t}=\frac{v}{U_{\infty}^{3}} \frac{d u_{\infty}}{d t}+\frac{v}{U_{\infty}^{2}} \frac{d u_{\infty}}{d x}
$$

Critical values of this parameter (when $d u / d t=0$ ) indicates the onset of re-laminarization. In original papers (Launder and Jones 1969, Jones and Launder 1972), there is a difference between the moderate and extreme pressure gradient; however, they claimed that for the values of $k>10^{-6}$ the structure of the turbulent boundary layer altered. According to the experiments, significant re-laminarization effects emerge when $k$ exceeds $2 \times 10^{-6}$, but a degeneration to laminar flow for the value of $k \approx 3 \times 10^{-6}$.

The present study aimed to examine the acceleration parameter and observe whether the above parameters are valid in the current situation or not.
Fig. illustrates the $k$ parameter variation along the wall between 5 percent of the acceleration period to the end of it for the imposed accelerations. As the first term $\left(\frac{\vartheta}{U_{\infty}^{3}} \frac{d u_{\infty}}{d t}\right)$ is smaller than the second one $\left(\frac{\vartheta}{U_{\infty}^{2}} \frac{d u_{\infty}}{d x}\right)$, as the acceleration is applied (when $\frac{d u_{\infty}}{d x}$ is more significant), the first term becomes negligible; however, the times after $0.5 \mathrm{~T}$, this alteration is declined, and the $K$ parameter becomes close to each other at each distinct time step. In both accelerations, the condition of $K_{t}>3 \times 10^{-6}$ to the occurrence of the re-laminarization phenomenon is valid in the early moments, so the incidence of this phenomenon is feasible

A specific location $x=0.7$ is selected to track the exact $K_{t}$ at which streamwise fluctuations reduce, and the history of this parameter is represented in Fig. 10. As the higher momentum rate causes more delay in the accelerated flow, the AC4 case inverses to laminar state in the higher value of $\mathrm{t}^{*}$ than the $\mathrm{AC} 3$ case.

However, what is significant in the comparison between those histories is that the value of $\mathrm{C}_{\mathrm{f}}$ in both cases starts to decline (Fig. 5) at an almost same value of $K_{t}\left(\cong 0.28 \times 10^{-6}\right.$ (Fig. 10-b)). Although acceleration affects fluctuating velocities and their correlation in a way that flow converts to the semilaminar state, the amount of imposed acceleration does not affect the re-laminarization mechanism significantly.

\subsection{Turbulence redistribution under FPG and APG}

The accelerated flow associates with a delay in turbulence propagation that plays a significant role in intense accelerations. The present manuscript tries to examine the pressure gradient effects on these delays in fluctuations development. As expected, FPG suppresses eddies and caused the formation of a semi-laminar boundary layer over that region. Actually, temporal acceleration and pressure reduction have approximately similar effects on the boundary layer structure regarding the pressure fluctuation reduction. The decrease of the pressure fluctuations initiates a decline in energy redistribution, all of which are caused by the great imposed momentum force in the outer layer. Pressure reduction initiates instability in the flow, which not only disturbs eddies shape but also affects turbulence production to not synchronize well with the outer flow Reynolds number. However, APG can mitigate the temporal acceleration effects that make the flow more orderly and stable.

The history of turbulence intensity in various streamwise positions is illustrated in Fig. . Similar to the zero pressure gradient case, turbulence intensity reduces to the least values in more intense acceleration; also, energy redistribution becomes weaker. In this figure, two regimes of flow are displayed distinctively: semi-laminar state and turbulent state, those refer to the before and after the minimum local $C_{f}$, (Fig. 5) respectively. 

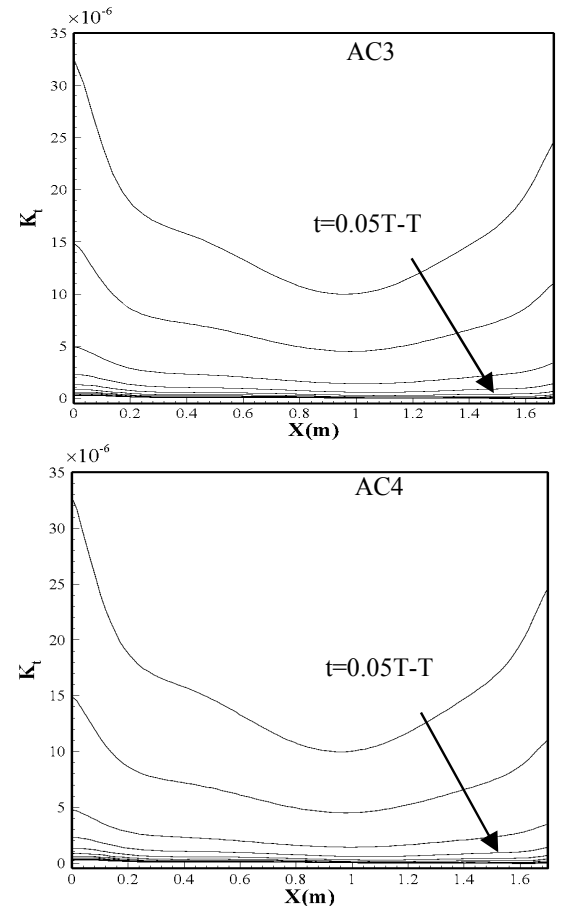

Fig. 9. Variation of acceleration parameter (K_t) during the acceleration period along the plate.

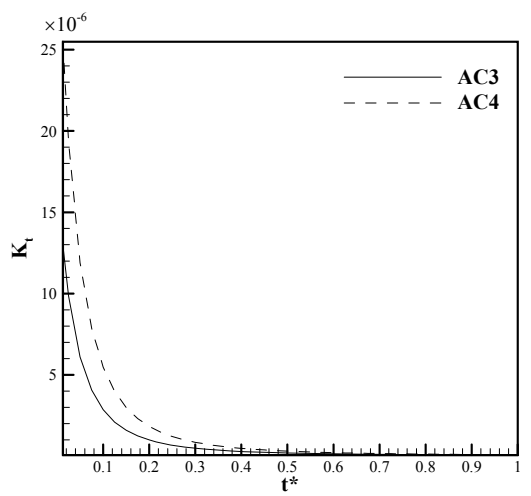

(a)

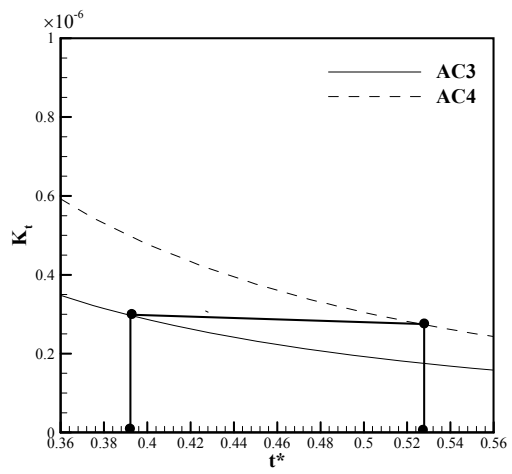

(b)

Fig. 10. Comparison of the acceleration parameter $\left(\mathrm{K}_{-} \mathrm{t}\right)$ during the acceleration period at $x=0.7 \mathrm{~m}$ for $\mathrm{AC} 3$ and $\mathrm{AC} 4$ cases.
At $x=0.6 \mathrm{~m}$, fluctuations freeze to such an extent that it can be assumed they almost decay. After that period, fluctuations abruptly grow, and disturbances transported from the near-wall to the upper layers initiate the bypass transition. During the freezing period (green), the increase of the flow Reynolds number in the outer layer makes the flow potential to govern a transition to the fully turbulent state. As the flow becomes enough potential, fluctuations start to grow and prompt the bypass transition in the boundary layer to burst the latent energy of the flow.

During the laminarization period, at positions with lower pressure (i.e., $x=0.7)$, the near-wall region is affected by the downstream flow (APG region). It starts to develop the fluctuations before the end of the delay period. Nevertheless, after a while, these fluctuations decline more severely and make the flow almost laminar. Although the APG region disturbances can distress their upstream, the Reynolds stress correlation is strongly affected in the FPG zone. In the case of sharper acceleration, after the re-laminarization period, fluctuations develop along the streamwise rather than the wall-normal direction, which implies that imposed temporal acceleration damped the wall-normal fluctuations ( $\left.v^{\prime}\right)$ more severely than the streamwise one $\left(u^{\prime}\right)$. Moreover, as the pressure reduces, the maximum acceleration parameter $\left(K_{t}\right)$ at which the flow starts to overthrow the fluctuations increases, so the flow becomes more unstable and the freezing period occurs more severely.

On the other hand, the imposed acceleration also affects the turbulence development in APG. Although the delay period is much shorter (almost half) and flow is more orderly, the freezing period is also significant under this condition, even though at the endpoints of the plate where momentum force is milder.

According to Fig., applied acceleration reduces the boundary layer thickness. Therefore, the concentration of turbulent fluctuations in areas close to the wall is increased compared to the upper layers. This relies on Reynolds stress correlation which is affected severely and decreases the diffusion rate in the wall-normal direction.

Generally, the fluctuation propagation in the FPG condition is lower than in the APG region. Although both zones witness damping in $u^{\prime}$ and $v^{\prime}$ developments, the development rate of the $v^{\prime}$ is much higher in APG condition. In other words, the Reynolds stress correlation is stabilized much more under the APG condition. Also, the turbulence intensity has consistent distribution in higher pressure fluctuations.

According to Fig. 12., turbulence kinetic energy develops with a different delay at distinct distances from the wall. The diffusion rate of disturbances in the APG conditions is more predictable and stable due to the lower level of the instabilities and imposed momentum. Alternatively, at the FPG region, Reynolds stresses and turbulence structures are exposed to the high instabilities of temporal and 
M. Kharghani and M. PasandidehFard / JAFM, Vol. 15, No. 2, pp. 311-324, 2022.
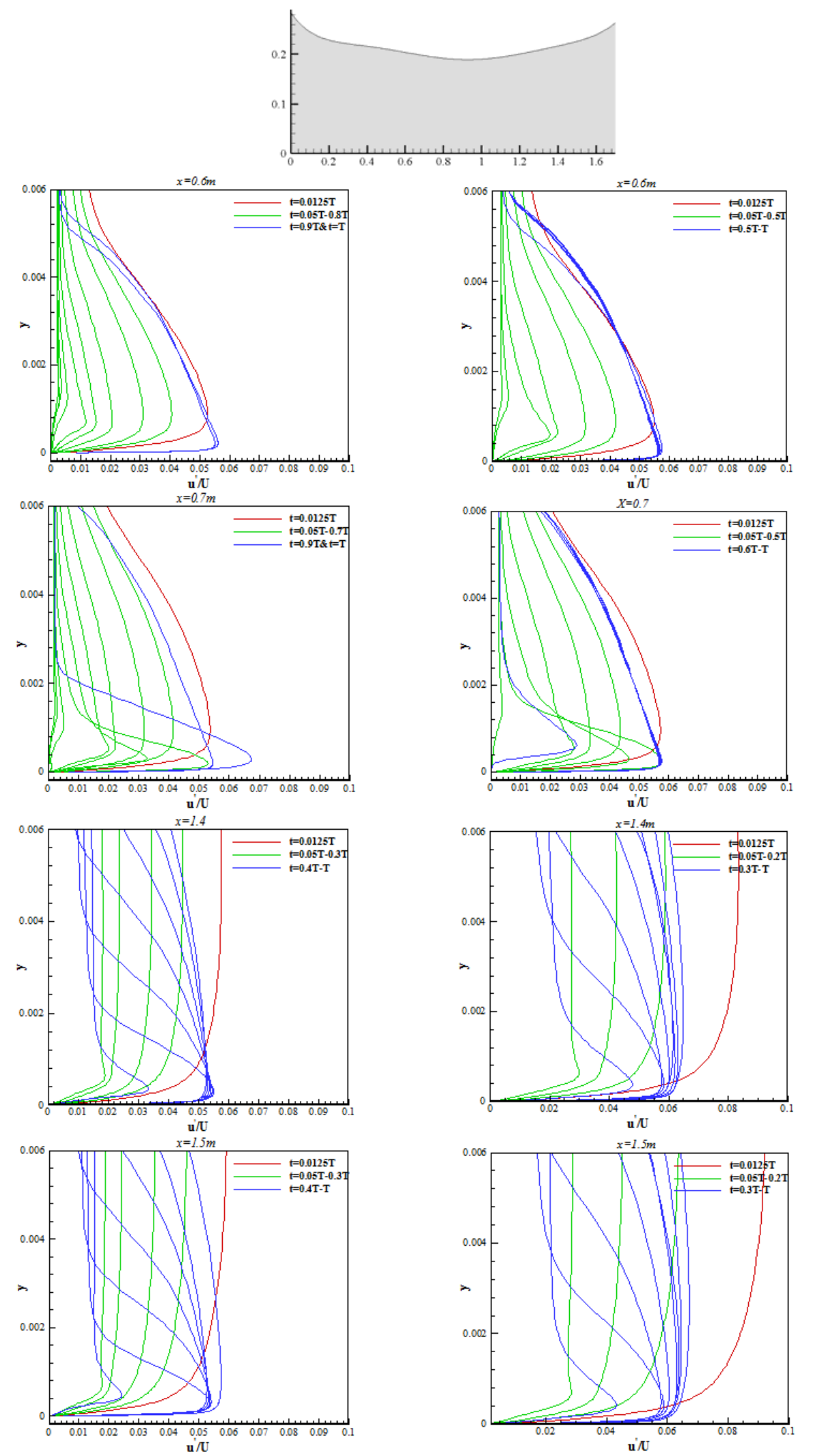

Fig. 11. Development of streamwise turbulence intensities history during laminarization (green) and transition (blue) periods at $x=1.0 \mathrm{~m}$. 

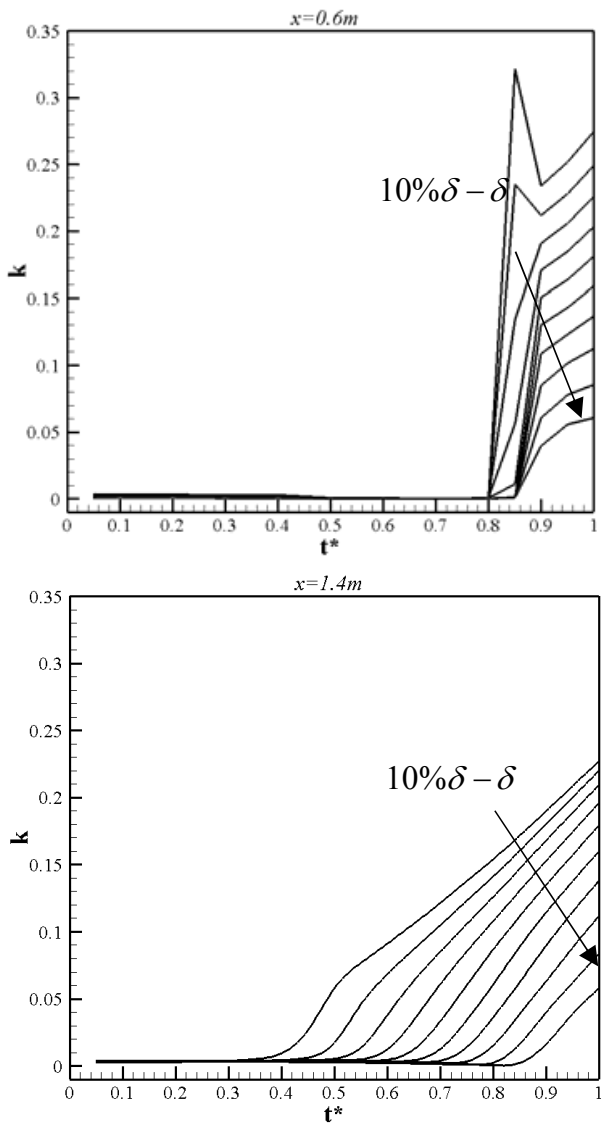

Fig. 12. Turbulence kinetic energy history of variation in different positions of the local boundary layer in $\mathrm{AC} 4$ case.

spatial acceleration that remarkably reduce the pressure fluctuations.

At $x=0.6 \mathrm{~m}$, the near-wall region $(10 \%$ and $20 \%$ of the local boundary layer thickness) witnesses a decrease in turbulence kinetic energy, even after the lag period. This implies that small eddies do not decay but freeze and save potential energy during the unconscious state. Indeed, the near-wall region is triggered more by instabilities and governs the bypass phenomena after the delay period. Another significant point is that the difference between delays from the near wall to the edge of the boundary layer is more significant in the APG than the FPG region. The reason relies on the boundary layer thickness and the higher $\mathrm{K}$ number at which re-laminarization occurred. Higher K numbers caused considerable damping of the fluctuations. However, after the consciousness of flow form variations, the diffusion rate becomes much greater than the APG to synchronize the inner layer turbulence structure with the outer layer Reynolds number.

\section{CONCLUSION}

The unsteady flowover the flat plate with non-zero pressure gradient condition is represented. The T3C5 test case is considered to examine both positive and negative pressure gradient conditions and their interactions with each other during the acceleration period. The following results are concluded:

- $\quad$ FPG affects the turbulent flow in the same manner as the temporal acceleration in damping the fluctuations. Therefore, the combination of these two conditions increases the delay in turbulence propagation. According to the decrease of the pressure fluctuations, the damped energy redistribution resulted in the local boundary layer thickness decline.

- Higher pressure fluctuations in the APG region amplifies the $R e_{\delta}$ which causes more freedom of fluctuations to redistribute and increase the turbulence production, Reynolds stress factors, and fluctuating velocity correlation stabilization, which caused the consistent distribution of turbulence intensity over the boundary layer.

- The near-wall region is triggered by instabilities and governs the bypass transition phenomena after the freezing period prior to the upper layers.

- The combination of spatially and temporal acceleration affects the velocity fluctuations correlation and makes their shape semi-elliptic. Higher imposed momentum can weaken the $v^{\prime}$ much more than $u^{\prime}$. Thus, as the flow becomes enough potential to retransmit to the turbulent state, streamwise fluctuations grow former and faster than the wall-normal fluctuations, which is the same scenario as the streamwise acceleration case.

- Regardless of the imposed momentum rate, viscous forces could overcome eddies and stabilize them in a specific $\mathrm{K}$ factor at a given pressure gradient.

- The re-laminarization led by imposed abrupt momentum is always followed by re-transition to the fully turbulent state even at the high accelerations. Thus, body fluxes may not be underestimated in such conditions because the outer layer Reynolds number governs a greater burst of energy after the freezing period.

\section{REFERENCES}

Bader, P., M. Pschernig, W. Sanz, J. Woisetschläger, F. Heitmeir, W. Meile and G. Brenn (2018). Experimental Investigation of Boundary Layer Relaminarization in Accelerated Flow. Journal of Fluids Engineering, Transactions of the ASME 140 (8).

Blackwelder, R. F. and L. S. G. Kovasznay (1972). Large-Scale Motion of a Turbulent Boundary Layer during Relaminarization. Journal of Fluid Mechanics 53 (1), 61-83.

Bourassa, C. and F. O. Thomas (2009). An Experimental Investigation of a Highly Accelerated Turbulent Boundary Layer. Journal of Fluid Mechanics 634, 359-404. 
M. Kharghani and M. PasandidehFard / JAFM, Vol. 15, No. 2, pp. 311-324, 2022.

Burnel, S., J. C. Raelison and J. M. Thomas (1990). Radial Distribution of the Reynolds Stress in a Turbulent Pulsating Flow in a Pipe. Elsevier. New York.

Escudier, M. P., A. Abdel-Hameed, M. W. Johnson and C. J. Sutcliffe (1998). Laminarisation and Re-Transition of a Turbulent Boundary Layer Subjected to Favourable Pressure Gradient. Experiments in Fluids 25 (5-6), 491-502.

Greenblatt, D. and E. A. Moss (2004). Rapid Temporal Acceleration of a Turbulent Pipe Flow. Journal of Fluid Mechanics 514, 6575.

He, K., M. Seddighi and S. He (2016). DNS Study of a Pipe Flow Following a Step Increase in Flow Rate. International Journal of Heat and Fluid Flow 57, 130-41.

He, S., C. Ariyaratne and A. E. Vardy (2008). A Computational Study of Wall Friction and Turbulence Dynamics in Accelerating Pipe Flows. Computers and Fluids 37 (6), 674-89.

He, S., C. Ariyaratne and A. E. Vardy (2011). Wall Shear Stress in Accelerating Turbulent Pipe Flow. Journal of Fluid Mechanics 685: 44060.

He, S. and J. D. Jackson (2000). A Study of Turbulence under Conditions of Transient Flow in a Pipe. Journal of Fluid Mechanics 408, 1-38.

He, S. and M. Seddighi (2013). Turbulence in Transient Channel Flow. Journal of Fluid Mechanics 715, 60-102.

He, S. and M. Seddighi (2015). Transition of Transient Channel Flow after a Change in Reynolds Number. Journal of Fluid Mechanics 764: 395-427.

Ichimiya, N. and F. FUJII. (1986). Properties in a Relaminarizing Turbulent Boundary Layer under a Favorable Pressure Gradient. Chemical and Pharmaceutical Bulletin 34 (1), 430-33.

Jones, W. P. and B. E. Launder (1972). Some Properties of Sink-Flow Turbulent Boundary Layers. Journal of Fluid Mechanics 56 (2), 337-51.

Jung, Se. Y. and Y. M. Chung(2009). Large-Eddy Simulation of Accelerated Turbulent Flow in a Pipe. 6th International Symposium on Turbulence and Shear Flow Phenomena, TSFP 2009 2009-June, 277-82.

Jung, S. Y. and K. Kim (2017). Transient Behaviors of Wall Turbulence in Temporally Accelerating Channel Flows. International Journal of Heat and Fluid Flow 67, 13-26.

Khaleghi, A., M. Pasandideh-Fard, M. MalekJafarian and Y. M. Chung. (2010). Assessment of Common Turbulence Models under Conditions of Temporal Acceleration in a Pipe. Journal of Applied Fluid Mechanics 3
(1), 25-33.

Launder, B. E. and W. P. Jones (1969). Sink Flow Turbulent Boundary Layers. Journal of Fluid Mechanics 38 (4), 817-31.

Mathur, A., S. Gorji, S. He, M. Seddighi, A. E. Vardy, T. O'Donoghue and D. Pokrajac (2018). Temporal Acceleration of a Turbulent Channel Flow. Journal of Fluid Mechanics $835,471-90$.

McEligot, D. M. (1983). Laterally Converging Flow. Part 2. Temporal Wall Shear Stress. Journal of Fluid Mechanics 127: 403-28.

McEligot, D. M., R. S. Brodkey and H. Eckelmann (2009). Laterally Converging Duct Flows. Part 4. Temporal Behaviour in the Viscous Layer. Journal of Fluid Mechanics 634, 43361.

McEligot, D. M. and H. Eckelmann (2006). Laterally Converging Duct Flows. Part 3. Mean Turbulence Structure in the Viscous Layer. Journal of Fluid Mechanics 549, 25-59.

Menter, F. R. (1994). Two-Equation Eddy-Viscosity Turbulence Models for Engineering Applications. AIAA Journal 32 (8), 15981605.

Menter, F. R., P. E. Smirnov, T. Liu and R. Avancha (2015). A One-Equation Local CorrelationBased Transition Model. Flow, Turbulence and Combustion 95 (4), 583-619.

Mizushina, T., T. Maruyama and H. Hirasawa (1975). Structure of the Turbulence in Pulsating Pipe Flows. Journal of Chemical Engineering of Japan 8 (3), 210-16.

Narasimha, R. and K. R. Sreenivasan (1973). Relaminarization in Highly Accelerated Turbulent Boundary Layers. Journal of Fluid Mechanics 61 (3), 417-47.

Narayanan, M., A Badri and V. Ramjee (1969). On the Criteria for Reverse Transition in a TwoDimensional Boundary Layer Flow. Journal of Fluid Mechanics 35 (2), 225-41.

Freymuth, P. (1985). The Vortex Patterns of Dynamic Separation: A Parametric and Comparative Study. Progress in Aerospace Sciences 22(3), 161-208.

Piomelli, U. and J. Yuan (2013). Numerical Simulations of Spatially Developing, Accelerating Boundary Layers. Physics of Fluids 25 (10).

Saavedra, J., J. Poggie and G. Paniagua (2020). Response of a Turbulent Boundary Layer to Rapid Freestream Acceleration. Physics of Fluids 32 (4).

Sarpkaya, T. (1991). Nonimpulsively Started Steady Flow about a Circular Cylinder. AIAA Journal 29 (8), 1283-89.

Scotti, A. and U. Piomelli (2002). Turbulence Models in Pulsating Flows. AIAA Journal 40 
M. Kharghani and M. PasandidehFard / JAFM, Vol. 15, No. 2, pp. 311-324, 2022.

\section{(3), 537-44.}

Sengupta, T. K., T. T. Lim, Sharanappa V. Sajjan, S Ganesh and J. Soria (2007). Accelerated Flow Past a Symmetric Aerofoil: Experiments and Computations. Journal of Fluid Mechanics 591, 255-88.

Shemer L. and E. Kit (1984). An Experimental Investigation of the Quasisteady Turbulent Pulsating Flow in a Pipe. Physics of Fluids 27 (1), 72-76.

Shemer, L., I. Wygnanski and E. Kit (1985). Pulsating Flow in a Pipe. Journal of Fluid Mechanics 153 (April), 313-37.

Soria, J. (2003). Multigrid CCDPIV Measurements of Accelerated Flow Past an Airfoil at an Angle of Attack of 30 " " 27: 667-76.

Spalart, P. R. (1986). Numerical Study of Sink-Flow Boundary Layers. Journal of Fluid Mechanics 172, 307-28.

Stefes, B. and H. H. Fernholz (1998). TThe Effects of a Favourable Pressure Gradient and of the
Reynolds Number on an Incompressible Axisymmetric Turbulent Boundary Layer. Part 2. The Boundary Layer with Relaminarization By. European Journal of Mechanics, B/Fluids 24 (2), 167-87.

Völker, F. R., R. Menter and S. Langtry (2006). Transition Modelling for General Purpose CFD Codes, no. March: 277-303.

Warnack, D. and H. H. Fernholz (1998). The Effects of a Favourable Pressure Gradient and of the Reynolds Number on an Incompressible Axisymmetric Turbulent Boundary Layer. Part 2. The Boundary Layer with Relaminarization. Journal of Fluid Mechanics 359, S0022112097008501.

Yuan, J. and U. Piomelli (2015). Numerical Simulation of a Spatially Developing Accelerating Boundary Layer over Roughness. Journal of Fluid Mechanics 780, 192-214. 